\title{
Agronegocios
}

\section{Artículo Costos de transacción económica: análisis de una experiencia real de venta en línea de alimentos}

Transaction cost economics: analysis of an online experience of food sales.

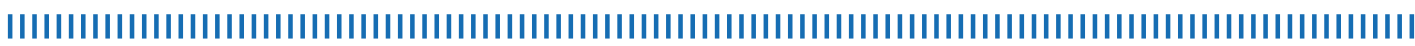

\section{Alexis Villalobos Monge ${ }^{1}$ Adriana Chacón Cascante ${ }^{2}$ Mercedes Montero Vega ${ }^{3}$ Olman Quirós Madrigal ${ }^{4}$}

Fecha de recepción: 08 de abril, 2020 Fecha de aprobación: 05 de junio, 2020 Vol. $6 \mathrm{~N}^{\circ} 2$ Julio- diciembre 2020

Villalobos, A., Chacón, A., Montero, V., y Quirós, O..(2020). Costos de transacción económica: análisis de una experiencia real de venta en línea de alimentos. Revista e-Agronegocios, 6(2). https://revistas.tec.ac.cr/index.php/ eagronegocios/article/view/5214

DOI: https://doi.org/10.18845/ea.v6i2.5214

${ }^{1}$ Universidad de Costa Rica. Costa Rica. Docente en la Escuela de Economía Agrícola y Agronegocios. Correo electrónico: alexis.villalobosmonge@ucr.ac.cr

2 Universidad de Costa Rica. Costa Rica.Docente en la Escuela de Economía Agrícola y Agronegocios. Correo electrónico: adriana.chaconcascante@ucr.ac.cr ${ }^{2}$

Universidad de Costa Rica. Costa Rica.Docente en la Escuela de Economía Agrícola y Agronegocios. Correo electrónico: mercedes.montero@ucr.ac.cr. 3 Universidad de Costa Rica. Costa Rica.Docente en la Escuela de Economía Agricola y Agronegocios. Correo electrónico: mercedes.montero@ucr.ac.cr.

4 Universidad de Costa Rica. Costa Rica.Docente en la Escuela de Economía Agrícola y Agronegocios. Correo electrónico: olman.quiros@ucr.ac.cr 


\section{Resumen}

El objetivo de este artículo es estimar la percepción del tiempo invertido en compras de alimentos por Internet, esto para aproximar los costos de transacción económica en este tipo de intercambios. También se evaluaron las variables predictoras de la respuesta del consumidor a la variable tiempo y su nivel de asociación con los costos de transacción. Se comparó la percepción del tiempo empleado en la compra de alimentos por canales diversos como son las ferias del agricultor, los supermercados y en línea, con el tiempo efectivo de esas transacciones. Los análisis se basan en la teoría del Estímulo-Organismo-Respuesta ( $\mathrm{S}-\mathrm{O}-\mathrm{R}$ del inglés Stimulus-Organism-Response) a través del uso de ecuaciones estructurales. Este estudio de caso generó los datos a partir de 223 encuestas a personas que realizaron compras de alimentos en línea por el portal de productos agroalimentarios de la Universidad de Costa Rica durante el periodo febrero-noviembre de 2019. Se logró determinar que la percepción del tiempo para realizar compras de alimentos por Internet es superior al tiempo que realmente se necesita. También fue posible verificar que las variables de tipo hedónicas (agrado al visitar el sitio web así como el tiempo invertido en la compra) tienen una fuerte relación con la percepción de los costos de transacción que deberá tomar en cuenta el consumidor de alimentos. Se presentan las conclusiones para su aplicación metodológica tanto desde un punto de vista académico como empresarial.

\section{Abstract}

The objective of this article is to estimate the perception of the time invested in food purchases over the Internet, as an approximation of transaction costs. The predictor variables of the consumer's response to the time perception and its level of association with transaction costs are also evaluated. The perception of time spent through different channels such as farmers markets, supermarkets and online was compared with the effective time of these transactions. The information is based on the Stimulus-Organism-Response theory (S-O-R) through the use of structural equations. These case study took the data from 223 surveys of people who made online food purchases through the agrifood products portal of the University of Costa Rica during the period February-November 2019, it was possible to find that the perception of time to buy food online is longer than it really takes. It was also possible to verify that the hedonic variables (pleasure when visiting the website as well as the time invested in the purchase) have a strong relationship with the perception of transaction costs that the food consumer must take into account. The conclusions are presented for its methodological application both from an academic and business point of view.

Key words: Transaction cost economics, electronic commerce, structural equation models, food

Palabras clave: Costos de transacción económica, comercio electrónico, modelos de ecuaciones estructurales, alimentos. 


\section{Agradecimientos}

Los autores agradecen el apoyo de la Vicerrectoría de Acción Social de la Universidad de Costa Rica por mantener la vigencia del proyecto Agroferia UCR durante el periodo de este estudio.

\section{Introducción}

Evaluar los costos directos e indirectos asociados a las transacciones de compra de bienes y servicios representa un importante reto para el consumidor, especialmente si se considera que ésta es una variable determinante en las decisiones de consumo, como lo asume la teoría tradicional (Coase, 1937; Williamson, 1989).

Los estudios sobre economía de costos de transacción (TCE del inglés Transaction Cost Economics) han incluido en los últimos años el comercio electrónico (EC por sus siglas en inglés Electronic Commerce), producto del terreno que han ganado como canal de comercialización.

A finales del siglo $X X$, se realizaron los primeros estudios empíricos sobre EC, especialmente enfocados en la identificación de productos con potencial de comercialización en medios virtuales como libros, zapatos o flores (Liang \& Huang, 1998). En el 2005 Teo \& Yu (2005), ampliaron los modelos con la inclusión de variables como incertidumbre, costos de transacción y confianza del consumidor. En el 2013, Kacen et al., (2013) incluyeron por primera vez la categoría alimentos en un análisis sobre el potencial del EC en la comercialización de estos bienes en comparación a medios tradicionales como supermercados. El estudio concluyó que los consumidores estarían dispuestos a pagar $21 \%$ menos por productos en medios virtuales que a través de canales tradicionales.

También se ha analizado otras variables relacionadas a los TCE de compras de bienes y servicios en línea. Por ejemplo, medios de pago digital versus efectivo (Wright et al., 2017); o el costo de políticas de devolución de productos (Li et al., 2019). A nivel de empresa también se han estudiado los TCE asociados a la implementación de alianzas estratégicas empresariales (Arslan et al., 2019), o al tiempo necesario para realizar compras de acciones bursátiles (Li \& Lam, 2002). No obstante, aún existen vacíos de literatura importantes relacionados al impacto de los TCE en el EC.

Es necesario ampliar el conocimiento sobre el impacto de los costos de transacción en la demanda de alimentos, específicamente nos interesa analizar las respuestas de los consumidores a la variable tiempo asociado a la compra de alimentos por medios electrónicos. Esta investigación proporciona nueva evidencia sobre la percepción temporal de las personas respecto a las compras de alimentos por diferentes medios, incluido el EC.

El objetivo principal del estudio fue analizar, empleando el estudio de un caso particular, las variables asociadas a los costos de transacción en que incurre el consumidor de alimentos 
al realizar compras por medios virtuales. Como complemento se analizan las diferencias de percepción de las personas sobre el tiempo necesario para efectuar la compra de alimentos por esta vía.

El trabajo se organiza de la siguiente manera: en la Sección 2 se detalla el referente teórico, haciendo énfasis en el modelo propuesto. En la Sección 3 se presentan los resultados obtenidos y en la Sección 4 se discuten los mismos. Finalmente, se presentan las conclusiones para su aplicación tanto desde un punto de vista académico como empresarial.

El modelo Estímulo-Organismo-Respuesta (S-O-R del inglés Stimulus-Organism-Response) es la base teórica empleada en la diferentes estudios sobre EC (6 de 34 estudios analizados). Chan et al., (2017) indica que éste es el enfoque preferido por diversos equipos de investigación que trabajan en EC. La teoría S-O-R parte del concepto clásico empleado en la psicología ambiental que asume que los individuos producen diferentes respuestas a fenómenos a partir de estímulos determinados (Chan, Cheung, \& Lee, op.cit.).

Para este estudio se utilizó como base la teoría S-O-R a través de un modelo que evalúa la demanda de alimentos por medios electrónicos. Se asume una relación causal entre variables exógenas (como la confianza y los impulsos hedónicos) y endógenas (la percepción sobre el tiempo empleado en las transacciones de compra) y la respuesta del consumidor (Figura 1).

El modelo permite predecir las respuestas a impulsos hedónicos, la confianza sobre la respuesta del consumidor hacia la compra de alimentos por EC, y aproximar la percepción del consumidor sobre la información relacionada con la búsqueda, adaptación y monitoreo de información. Se consideraron los siguientes estímulos:

a) La confianza en las compras en línea. Diversos estudios (Liao et al., 2011; Martínez-López et al., 2014: Pavlou, 2003) resaltan que la confianza de los consumidores es un estímulo importante al realizar compras en línea. Se consideran dentro de esta categoría de estímulos la familiaridad que el potencial comprador tendrá con el sitio de EC así como el método de pago. Gefen (2000) por ejemplo realizó una mezcla de ambos enfoques. Para evaluar la confianza se definieron como variables: 1) la confianza en los comentarios de otros compradores (CF2), 2) la confianza en la promesa de calidad ofrecida por el sitio web (CF3), 3) la confianza por la información del productor ofrecida por el sitio web (CF4), 4) la confianza en las sugerencias que se puedan recibir del sitio de EC para apoyar la compra de alimentos (CF5).

b) La percepción del tiempo necesario para realizar la compra de alimentos y su relación con los TCE implícitos en el proceso de compras en línea versus los efectuados en los canal es actuales seguidos por el consumidor. Autores como Williamson (1989) y Teo \& Yu (2005) plantean que los consumidores siguen una lógica racional al considerar los costos en que debe incurrir para apropiarse de diversos bienes y servicios. En ese sentido, se estarán tomando en consideración la percepción del tiempo que necesitan los consumidores para adquirir bienes agroalimentarios por diferentes vías y se estarán comparando con aquellos asociados 
por emplear EC, de ahí que las variables elegidas para este fin fueron: 1) Percepción del tiempo de búsqueda para realizar la compra $\left(\mathrm{TC}_{1}\right)$, 2) Percepción del tiempo de monitoreo para realizar la compra (TC2)y 3) Percepción del tiempo de adaptación para realizar la compra (TC3).

c) El placer de comprar en línea. Como parte de los estímulos que se toman al momento de entender las reflexiones que las personas consideran en las decisiones de compra, las de naturaleza hedónica complementan aquellas de tipo utilitario. En los estímulos a) y b) se toman variables para evaluar las ligadas a este último tipo de conducta utilitarista. Para las variables hedónicas o asociadas con comportamientos explicados por el placer de comprar por EC, se sigue lo propuesto por autores que han buscado entender la manera en que las personas realizan compras particularmente por el placer que ello les provoca (Cai \& Xu, 2006; Fang et al., 2016), con ello se busca manejar los dos tipos de comportamientos que podrían influir en la decisión final de compra. Por esto, se definieron como variables: 1) El tiempo empleado en el sitio de comercio electrónico fue realmente agradable (HD1), 2) Placer de utilizar este sitio de comercio electrónico no solo por los artículos que se puede comprar (HD2) y 3) El agrado de visitar el sitio de EC (HD3).

d) La respuesta del consumidor sobre la compra de alimentos en línea. Se asume la existencia de una relación positiva entre las variables latentes "confianza" e "impulsos hedónicos" así como una relación negativa por parte de los TCE y la respuesta final de compra. Estas relaciones se estarían presentando para la compra de bienes y el nivel de gasto, así como las intenciones futuras de compra por esta vía, además de la satisfacción en general de emplear EC. Este último supuesto partirá de lo identificado por Floh \& Madlberger (2013) respecto a la satisfacción de realizar compras en línea. La motivación asociada a la hipótesis de los TCE sigue la línea del trabajo de Teo \& Yu (2005). Se definieron como variables observables para este estímulo: 1) la probabilidad futura de realizar compras de alimentos en línea (R1), 2) la satisfacción general del consumidor por la experiencia de compra (R2) así como 3) la percepción del gasto de alimentos realizado en línea respecto al consumo habitual que presenta la persona por este tipo de bienes (R3).

Se plantearon las siguientes hipótesis en el trabajo:

H1: "La confianza al comprar bienes agroalimentarios por EC está positivamente relacionada con la respuesta general de compra del consumidor".

H2: "Los impulsos hedónicos al comprar bienes agroalimentarios por EC están positivamente relacionados con la respuesta general de compra del consumidor".

H3: "La percepción de los costos de transacción económica al comprar bienes agroalimentarios por EC están negativamente relacionados con la respuesta general de compra del consumidor". 


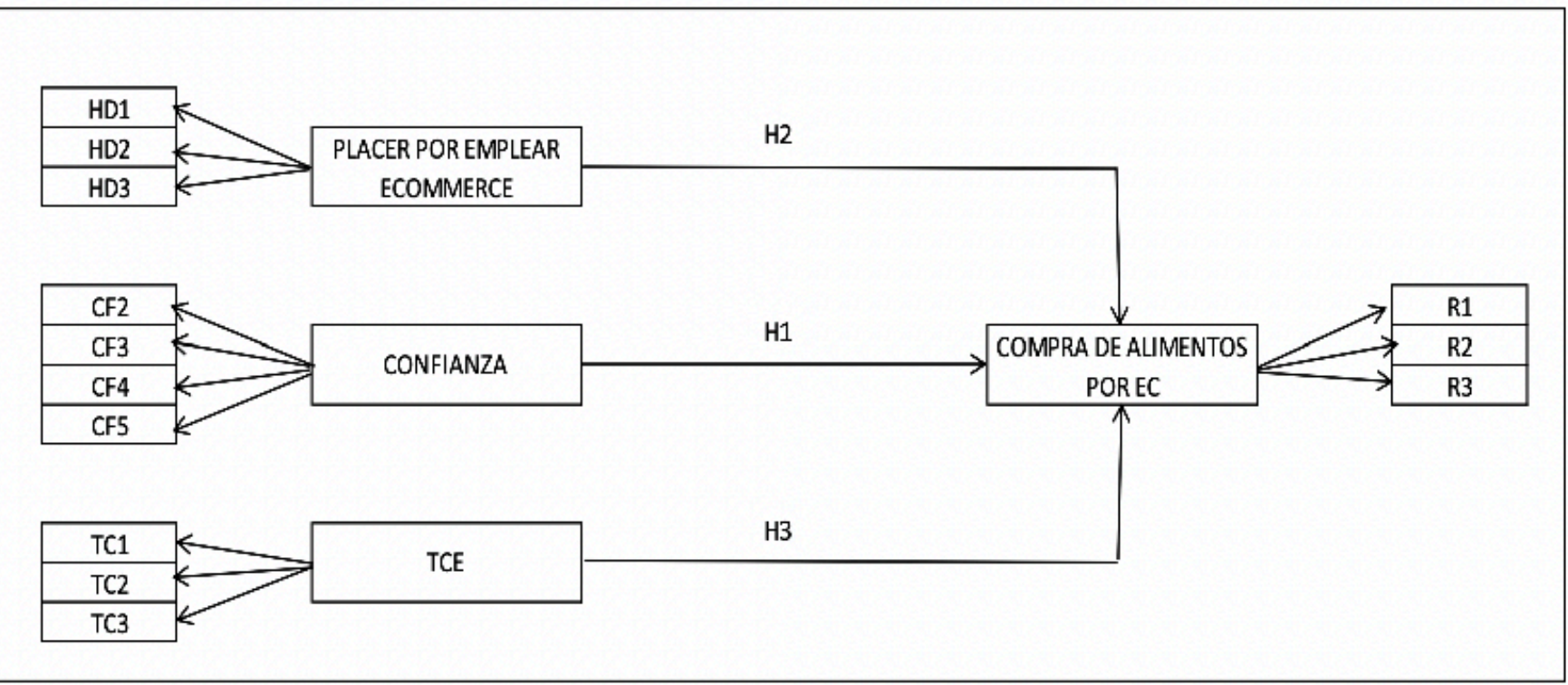

Figura 1. Modelo S-O-R para las variables asociadas con la confianza, los impulsos hedónicos y la percepción de los TCE al momento de comprar alimentos por medios virtuales.

Como se indicó anteriormente, el supuesto implícito en los 3 casos es la existencia de una relación entre los "estímulos" y la variable compra de alimentos por EC. Se usa el modelaje econométrico a partir de ecuaciones estructurales (o SEM en inglés por Structural Equation Models) tal como lo hicieron otros autores como (Doong \& Wang, 2011; Kim, Galliers, Shin, Ryoo, \& Kim, 2012; Kim, Xu, \& Gupta, 2012; Liao, Liu, \& Chen, 2011; Lin, Lu, Wang, \& Wei, 2011; Martínez-López, Pla-García, Gázquez-Abad, \& Rodríguez-Ardura, 2014; Schierz, Schilke, \& Wirtz, 2010).

Los SEM implican la combinación de varias técnicas estadisticas, considerando particularmente el análisis de la estructura de covarianza de la información (Kline, R., 2011). El planteamiento teórico base asume la siguiente relación:

$\operatorname{cov} X Y=r X Y S D X S D Y$

Donde covXY representa la fuerza entre la asociación existente de la variabilidad del conjunto de datos $X$ y $Y$. Esta relación se obtiene del producto del coeficiente de correlación de Pearson ( $r X Y$ ) y las respectivas desviaciones estándar de ambos grupos de datos. Kline (op. cit) anota el riesgo de confundir los SEM de forma análoga a la realización de un análisis de varianza (o ANOVA en inglés por Analysis of Variance); sin embargo, aclara que la diferencia entre ambas herramientas radica en que el análisis de las medias en SEM se efectúa de forma más amplia del que se logra realizar en un ANOVA, ya que en el primero se logra precisar dicho análisis para variables latentes o no observables mientras que en la segunda técnica ello no es factible de realizar. Esta diferencia apunta a que en SEM es posible obtener tanto una estructura de covarianzas como una estructura de medias, y esta última permite el cálculo 
de parámetros de la media, lo que es una condición sine quo non para efectuar el análisis de la información entre datos pertenecientes a diferentes grupos de personas, ello porque esta técnica emplea análisis de regresiones multivariadas.

Los SEM emplean el análisis de senda para descomponer los efectos que provoca una variable sobre otra de acuerdo a su causalidad sea total, directa e indirecta (Bollen, 1987). En este análisis, se establecen relaciones entre variables de la forma:

$$
\eta=B \eta+\lceil\xi+\zeta
$$

Donde $\eta$ es un vector $\mathrm{m} \times 1$ de variables latentes (o no observables) endógenas, B es una matriz $\mathrm{m} \times \mathrm{m}$ de coeficientes vinculados con las variables $\eta$, $\xi$ es un vector $\mathrm{n} \times 1$ de variables latentes exógenas, $\Gamma$ es una matriz de coeficientes $\mathrm{m} \times \mathrm{n}$ relacionando a $\Gamma$ con $\eta \mathrm{y} \zeta$ es un vector $\mathrm{m} \times 1$ de los errores en la ecuación. Las ecuaciones que permiten determinar (2) se expresan en la forma:

$$
\begin{array}{r}
x=\Lambda_{x} \xi+\delta \\
y=\Lambda_{y} \mathrm{\eta}+\varepsilon
\end{array}
$$

Donde xyydenotan variables observables (las respuestas a las preguntas planteadas por el investigador), $\Lambda_{x}$ es una matriz de coeficientes $q \times n$ que relacionan $x$ con $\xi$, $\wedge_{y}$ es una matriz de coeficientes de los efectos de $\eta$ en y de tamaño $\mathrm{p} \times \mathrm{m}$.

\section{Metodología}

Los datos se recolectaron a partir del diseño de un experimento utilizando el sitio web del proyecto Agroferia de la Universidad de Costa Rica (UCR)․․․․ proyecto Agroferia UCR es una iniciativa universitaria de la Facultad de Ciencias Agroalimentarias dirigida a la capacitación de diversos actores del sector agroalimentario, la generación de nuevos conocimientos y apoyar el proceso de enseñanza-aprendizaje en el tema de comercialización de bienes y servicios de origen agroalimentario. La recolección de datos se realizó entre febrero y noviembre de 2019.

El experimento consistió en la simulación de experiencias reales de compra de alimentos, las cuales generaron información en dos sentidos: a) Medición de tiempos de compra de alimentos en la base de datos del sitio web del proyecto Agroferia UCR, b) Participación de compradores que, voluntariamente, accedieron a completar una encuesta al momento de finalizar la compra efectuada por el sitio web. 
Las preguntas empleadas para este trabajo fueron valoradas empleando una escala Likert de uno a cinco (siendo tres el valor de indiferencia), empleando una escala desde "Muy en desacuerdo con la afirmación" hasta "Muy de acuerdo con la afirmación" .Se desarrolló una prueba piloto con docentes universitarios para revisar la relevancia de las preguntas y a partir de esos resultados fue establecida la versión final del cuestionario aplicado.

\section{Descripción del experimento realizado}

El experimento se planteó siguiendo el enfoque propuesto por Smith (1989), que postula que la economía debe ser un ejercicio de observación de la realidad y no uno basado en planteamientos teóricos. Se propusieron situaciones de compra de alimentos, con escenarios que permitieran a las personas participantes analizar sus decisiones e implicaciones como si estuvieran frente a una situación real.

Durante 30 sesiones semanales de venta se ofrecieron 171 productos de las siguientes tipologías: granos y semillas, café, vegetales, frutas frescas y productos agroalimentarios con algún nivel de procesamiento (miel de abeja, jugo de naranja, salsas, mermeladas). El acceso al sitio electrónico se encuentra limitado a funcionarios con nombramiento activo como empleados de la Universidad de Costa Rica. Cada persona que formó parte de esta población dispone de un identificador personal a nivel de bases de datos, el cual permite validar el acceso del funcionario a los diferentes sistemas informáticos que se encuentran bajo el ámbito de administración de la Universidad.

Para incentivar la participación en la encuesta, se ofreció como incentivo económico la participación en sorteos semanales de órdenes de compra por el sitio web del proyecto. A las personas que participaron en la consulta se les solicitó completar una única vez el cuestionario en línea, donde se recolectó información demográfica (edad, sexo, escolaridad, lugar de residencia, frecuencia de compra semanal para estos productos, frecuencia semanal de acceso a internet por motivos de ocio y trabajo, lugares acostumbrados para realizar la compra de estos bienes, ingreso mensual, tamaño de la familia). Adicionalmente a la información demográfica, se preguntó por la información relacionada con los estímulos que aparecen en la Figura 1. Este proceso de recolección de información (anónima) sigue la lógica mostrada por estudios previos realizados en el tema de EC (Floh \& Madlberger, 2013; C. Kim et al., 2012; Liao et al., 2011; Lin et al., 2011; Martínez-López et al., 2014).Otra variable que se consideró fue la época del año (seca o lluviosa) en que fue efectuada la compra, esto para revisar la existencia de diferencias en las respuestas entre los subgrupos demográficos empleados.

De una población de 421 personas que realizaron compras durante el periodo de estudio, un total de 273 completaron la encuesta ( $64,8 \%$ de la población total), lo que se asume como una cantidad de respuestas típicas para encuestas virtuales. Fueron personas de ambos sexos entre los 21 y 72 años de edad, las cuales compraron alimentos por el sitio web del proyecto Agroferia UCR. Luego de revisar la totalidad de encuestas, fueron desestimados 50 casos (18,3\% del total), quedando el tamaño de muestra finalmente en 223 encuestas válidas. En el 
Anexo 1 se observa la información demográfica general de la población encuestada.

Se destaca que la mayor parte de la población encuestada corresponde a mujeres, la edad promedio fue de 42 años con poca variación en las edades por género (media de hombres y de mujeres fue de 41 y 42 años respectivamente), la cantidad de personas con estudios universitarios fue considerable como era de esperarse (83.9\%), el tamaño de las familias concuerda con los datos a nivel nacional respecto a esta variable ya que los hogares costarricenses mantienen en promedio de 2 a 3 personas (INEC, 2019). Finalmente, el ingreso promedio de los encuestados concuerda con la información proporcionada por INEC (op.cit.) para las familias pertenecientes a los quintiles III, IV y V existentes en la sociedad costarricense (Anexo 1).

Con la información resultante, se llevaron a cabo dos evaluaciones de los componentes relacionados con los TCE en la compra de alimentos por EC: a) una primera evaluación donde se realizó el modelaje de ecuaciones estructurales, b) una segunda evaluación donde se tomó la percepción del tiempo en que incurren los encuestados, esto cada vez que realizan la compra de alimentos en cada uno de los canales que frecuentan para adquirir dichos bienes. Esta evaluación también comparó el tiempo que realmente emplearon los consumidores en las compras efectuadas durante el periodo del experimento en el sitio web del proyecto Agroferia UCR.

Para efectuar la primera evaluación asociada con las hipótesis $\mathrm{H}_{1}, \mathrm{H}_{2}$ y H3 , se utilizó el paquete Lavaan (Latent Variable Analysis por sus siglas en inglés) de R. En esta evaluación se realizó un Análisis Factorial Confirmatorio (CFA del inglés Confirmatory Factor Analysis) para evaluar la validez del modelo de medición así como el Alfa de Cronbach por medio del software SPSS ( $)$ versión 25 para comprobar la confiabilidad y/o consistencia de los datos obtenidos además de los resúmenes para la información demográfica de los encuestados. La segunda evaluación consistió en la percepción de los encuestados sobre el tiempo empleado por cada canal de comercialización utilizado para adquirir alimentos, esa información fue contrastada con los registros de la base de datos del proyecto Agroferia UCR del tiempo empleado en cada transacción durante el periodo de evaluación del experimento.

\section{Resultados}

Las condiciones de las personas que participaron en el estudio presentan diferencias significativas para las variables de agrupación. Para el caso de la edad, por época se observa una mayor edad promedio en hombres mientras que la edad promedio de las mujeres fue igual (42 años) tanto en época seca como lluviosa. Respecto a la escolaridad, se observan mayores diferencias para los participantes con estudios doctorales ya que el coeficiente de variación (CV) entre ambas difiere en 10.8\%. Para la categoria "Otros" la diferencia del CV fue aún mayor (16.3\% entre épocas).

Para las clasificaciones por ingreso, las diferencias entre los CV según época no presentan mayores diferencias en comparación a las otras categorias de agrupación (a excepción de las 
personas con doctorado como se comentó anteriormente). Para la clasificación de integrantes del núcleo familiar, las principales diferencias por época se observan para las personas que conviven con más cuatro personas (20.2\% de diferencia entre épocas).

\section{Estimación del modelo con los datos obtenidos}

La edad de la persona y la época (seca o lluviosa) en que fue efectuada la compra parecen ser las variables que agrupan de mejor manera los resultados respecto al modelo empleado (se discutirá más adelante), por lo que se muestra la información demográfica clasificada por dichas variables.

En la Figura 2 se muestra el diagrama de sendas con las relaciones directas determinadas entre las variables.

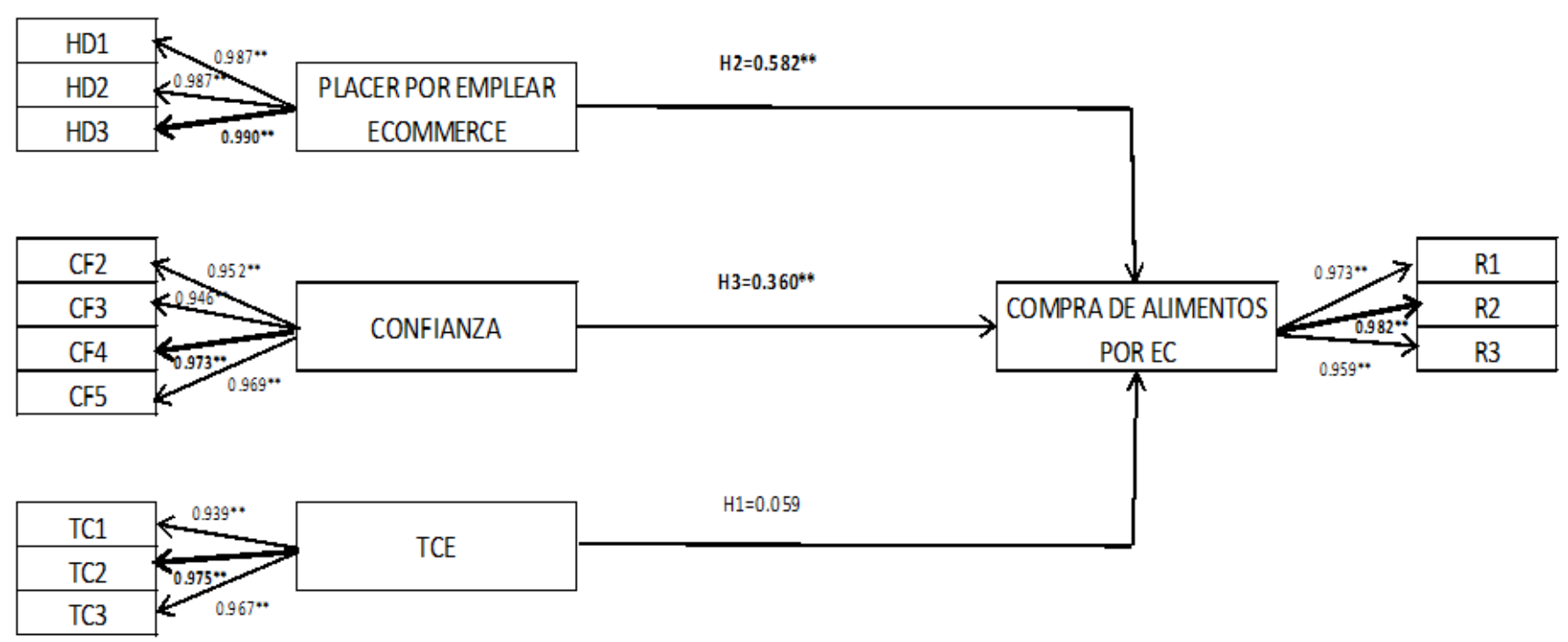

Figura 2. Modelo S-O-R para las variables asociadas con la confianza (CF), los impulsos hedónicos (HD) y la percepción de los costos de transacción (TCE) al momento de comprar alimentos por medios virtuales (R). (Negrita=Principal efecto directo, ${ }^{* *}=$ significancia 0.01)

Los efectos directos, indirectos y totales se muestran en el Anexo 2. Los resultados indican la existencia de efectos significativos para las variables latentes Confianza (CF) y para los impulsos hedónicos (HD), el cual presenta un efecto total importante para una de las variables respuesta (HD1). Para el caso de la variable TCE el modelo propuesto no logró determinar efectos totales con significancia estadistica menor al 5\%.

El principal efecto total se observa desde la variable asociada con el tiempo empleado por parte del consumidor en EC para adquirir alimentos. Los resultados indican que ante un nivel de aumento de una desviación estándar en la percepción de lo agradable del tiempo inverti- 
do en EC al comprar alimentos predice un aumento en la media de la respuesta general del consumidor de aproximadamente 1.6 veces, manteniendo de manera constante los valores de Confianza y TCE.

Los resultados del modelo tienen un alfa de Cronbach de 0.994 lo que indica que el instrumento es confiable. El CFA aplicado obtuvo parámetros donde el modelo es plausible para los datos empleados (CFI=0.966, $\mathrm{TLI}=0.955$, SRMR=0.019, RMSEA=0.120). El valor de $2=248.794$ fue significativo ( $\mathrm{p}$-value<0.05) lo cual podría deberse a que el tamaño de muestra N>200 genera este tipo de situaciones en modelos SEM para muestras grandes según lo indicado por varios autores (Kline, 2011 pp. 200-201; Ramlall, 2017 pp. 65).

Los resultados anteriores permiten aceptar las hipótesis H1:"La confianza al comprar bienes agroalimentarios por EC está positivamente relacionada con la respuesta general de compra del consumidor" y H2: "Los impulsos hedónicos al comprar bienes agroalimentarios por EC están positivamente relacionados con la respuesta general de compra del consumidor" pero no la H3: "La percepción de los costos de transacción económica al comprar bienes agroalimentarios por EC están negativamente relacionados con la respuesta general de compra del consumidor", cuyo nivel de significancia es mucho mayor al 1\% (0.368). Los resultados también sugieren que el principal efecto directo sobre la variable Respuesta (R) parece explicarse principalmente por la experiencia de compra, lo que es plausible de asociar con el resultado del mayor efecto que presenta la variable latente HD (0.582) sobre la variable respuesta de la compra de alimentos por EC.

El análisis de la matriz de covarianza obtenida entre las variables permite establecer, con una significancia del $5 \%$, que existe una fuerte relación positiva entre las mismas. La covarianza del constructo CF es mayor con HD (0.982) versus el que se predice para con TCE (0.963). La covarianza entre HD y TCE también es positiva (0.947). De esto se puede decir que el modelo predice una relación similar entre los cambios que puedan relacionarse entre las variables tomadas en consideración, no obstante que las variaciones que experimente el consumidor en los estímulos asociados con "Confianza" podría representar una mejor aproximación con los cambios que se pudieran producir en la percepción que este llegara a tener de los TCE.

Respecto a la capacidad predictiva del modelo sobre la percepción del consumidor sobre el tiempo, como variable relevante de los TCE, se debe indicar que los resultados del trabajo de campo no permiten confirmar con un nivel de significancia menor al 5\% dicha capacidad de predicción. Esto podría deberse a que el "efecto de aprendizaje" como lo denomina Liang (1998), aún es incipiente por lo reciente de este canal para comprar alimentos, de ahí que se presuma que las respuestas proporcionadas en el cuestionario a estas preguntas aún no han logrado alcanzar un nivel suficiente de conocimiento, sobre las ventajas y desventajas, que desde los TCE se deberán considerar al momento de efectuar compras de alimentos por medios virtuales. 


\section{Percepción del tiempo empleado por cada canal de compra de alimentos}

La percepción del tiempo que emplea el consumidor en realizar sus compras de alimentos según el canal especifico (ferias del agricultor, supermercados, sitios en internet) presenta diferentes valores. Los resultados se presentan de acuerdo al canal de preferencia indicado por cada persona en el Anexo 3.

Estos resultados tienen dos implicaciones importantes. En primer lugar, confirma que las ferias del agricultor han dejado de ser el principal canal para comprar alimentos frescos por parte de la mayoría de la población, esto es concordante con lo que han indicado varios autores sobre este tema (PIMA, 2017; Villalobos-Monge \& Sánchez-Chacón, 2013).

En segundo lugar, la percepción del tiempo empleado por compra varía significativamente porcada canal, esto puede sugerir que los consumidores no dan seguimiento preciso a esta variable, afectando los TCE percibidos por cada canal y sus decisiones sobre el canal a utilizar.

Las diferencias entre las medidas de posición obtenidas, muestran una mayor debilidad de los mismos (promedio y mediana) principalmente para las compras realizadas por Internet que por las efectuadas en ferias del agricultor y supermercados respectivamente, donde para este último caso los resultados sugieren que existe una mayor precisión en la percepción sobre el tiempo que se emplea durante cada compra efectuada.

Para contrastar los resultados obtenidos de los encuestados sobre el tiempo que se debe tomar en consideración al momento de efectuar una compra en línea, se realizó la revisión de las transacciones efectuadas durante el periodo de estudio en la base de datos del sitio Agroferia UCR y fue posible obtener la información que se detalla en el Anexo 4.

Los resultados en general muestran diferencias destacables entre la percepción que tienen los consumidores del tiempo que significa la compra en línea respecto a lo que en realidad les implica. El tiempo promedio empleado por cada compra fue de 4 minutos con 8 segundos, donde al comparar por sexo de la persona se observa una leve variación. Cabe destacar que el valor promedio es aproximadamente una tercera parte de la percepción de los encuestados donde además la revisión de los datos muestran que un $90.7 \%$ de las compras fueron realizadas en menos de 10 minutos, $78.1 \%$ tardó menos de 5 minutos y solamente el $1 \%$ empleó más de 30 minutos en la compra por este canal.

\section{Discusión}

El modelo propuesto permite identificar, por una parte, que los impulsos hedónicos impactan con mayor relevancia la respuesta del consumidor en comparación al efecto que provoca la confianza. Esto podría significar que los estímulos ligados a emociones placenteras asociadas, por ejemplo, con el tiempo dedicado a la compra de alimentos por medios virtuales (HD1) influirian más fuertemente en la percepción de los TCE que efectivamente existan al momento 
de comprar alimentos por EC. Esto supone que, aunque la percepción de dichos costos pareciera no estar claramente delineada para los consumidores, las acciones orientadas a mejorar los estímulos que el consumidor de alimentos pueda recibir desde dicho grupo de motivaciones (particularmente las asociadas con el agrado que la persona pueda sentir al visitar el sitio web) provocarian una mayor respuesta general, y por consiguiente, una diferencia más favorable entre dicha respuesta y los TCE implícitos para obtenerla.

Lo anterior no contradice lo expuesto por diversos autores respecto a la relevancia que ocupa la confianza como estímulo hacia los TCE por medios electrónicos, ello porque se comprueba la existencia de una relación positiva de la confianza en la respuesta general, donde al igual que lo afirmado por Teo \& Yu, (2005, pp 453.) se tiene certeza en que "el rol de la confianza en la teoría de los costos de transacción es importante porque, en algún sentido, reduce lla magnitud del estos costos".

El efecto que se logró determinar para la influencia que ocupa la confianza en la respuesta general del consumidor se puede catalogar como relativamente bajo $(<0.5)$ donde el mayor efecto directo es explicado por la disponibilidad de información del productor que el sitio de comercio electrónico logre evidenciar al consumidor (CF4), sin embargo todos los efectos directos que las variables observables provocan sobre la variable latente "Confianza" fueron muy similares entre sí, por ejemplo, la diferencia entre CF4 y CF5 (estímulo que proporciona la confianza en las sugerencia de compra proporcionadas por el sitio de EC) es pequeña no obstante que implican diferentes dimensiones de contenido y diseño del sitio. Este es un elemento que futuras investigaciones podrian abordar para profundizar en el tema.

Respecto a los principales estímulos de los TCE que se ven influenciados por la percepción del consumidor de alimentos, se debe indicar que la percepción de los costos de adaptación (TC2) constituye el efecto directo más significativo.

Adicionalmente, la existencia de un mayor efecto directo sobre la respuesta de compra generado por la satisfacción general que tuvo el consumidor por esta experiencia (R2) concuerda con el mayor efecto de la variable latente "Impulsos Hedónicos" (HD) que se obtuvo de la regresión sobre la variable general de respuesta. Lo anterior se asocia con resultados publicados por diversos autores (para otros tipos de bienes) al momento de analizar los comportamientos del consumidor por EC. Por ejemplo, en el trabajo de Floh \& Madlberger, (2013) se encontró que los impulsos de tipo hedónico tienen un mayor efecto sobre la compra en línea que otros estímulos, como la búsqueda de información, para el caso de libros.

Resulta interesante comparar las diferencias encontradas entre la percepción que tienen las personas del tiempo empleado en la compra de alimentos por diferentes canales y el valor exacto de compra por medios virtuales. El tiempo promedio y la mediana por compra muestran variaciones entre ambas medidas del $281 \%$ del tiempo que se percibe versus el tiempo real que le toma a la persona realizar una compra por EC para el primer caso y del $41 \%$ para el segundo caso respectivamente. Esto sugiere que la mediana en estos casos será la medida 
de posición más precisa para establecer esta percepción. A nuestro conocimiento no existen referencias previas en la literatura para este tipo de valores en EC.

Lo apuntado anteriormente, permite argumentar que los TCE que perciben las personas al comprar alimentos por EC no se tienen dimensionados en una escala precisa, lo cual dificulta la modelación de este comportamiento en virtud de que el tiempo es un elemento trascendental para entender la fluidez con que se dispone de nueva información para tomar decisiones de compra (simetría o asimetría entre los actores del mercado). Cuando Williamson (1989, pp. 136) indicaba que el planteamiento de los TCE implican un abordaje económico más autoconsciente de los supuestos conductuales, se podría añadir que el tiempo representa en EC un asunto de vital interés, y que para el caso de bienes como los alimentos, esta es una variable que los consumidores no manejan con suficiente precisión al momento de tomar la decisión de comprar por este canal.

Finalmente, la evidencia de que 9 de cada 10 compras de alimentos por Internet tardan menos de 10 minutos en ser efectuadas direcciona los resultados de esta investigación hacia aplicaciones en potenciales emprendimientos de esta naturaleza. Existen trabajos que han estudiado en EC la manera en que las sociedades humanas dimensionan la percepción del tiempo como parte de sus expresiones particulares (Xu-Priour et al., 2017) y, para paises americanos, ese estudio sugiere que la orientación "hacia el futuro" en cuanto a esta percepción temporal podría jugar un rol interesante en las estrategias orientadas a destacar las fortalezas de comprar alimentos por sitio de EC, donde el tiempo que podría tomar es menor en comparación a otros canales.

\section{Conclusiones}

De este trabajo se pueden concluir varios aspectos asociados con el análisis de los TCE y la realidad de los medios virtuales para comercializar alimentos. Es importante resaltar que los trabajos en EC han evolucionado en los últimos 20 años, donde los abordajes han mantenido cierto paralelismo con lo que se observa en la dinámica de canales de comercialización convencionales, y este estudio se formuló en esa misma línea.

En primer lugar, se comprueba que los impulsos hedónicos y la confianza pueden influir positivamente en la respuesta final del consumidor de alimentos, ello por la significativa relación existente entre estas variables de análisis. La existencia de factores superiores a 0.9 en la covarianza entre estas variables implica que al momento de considerar los costos asociados con el proceso de compra de alimentos en línea, generar motivaciones desde estos dos conjuntos de estímulos estarían prediciendo una respuesta positiva en la percepción de los TCE y la respuesta general del consumidor. Esto será de importancia para la práctica empresarial que se pueda llegar a relacionar con este tipo de actividad económica.

En segundo lugar, la precepción que el consumidor pueda tener sobre el tiempo necesario para buscar, monitorear y adaptar las compras de alimentos que sean efectuadas en línea son 
costos todavía imprecisos de dimensionar, ello por las considerables diferencias en las magnitudes existentes entre lo que realmente ocurre en comparación a esta percepción temporal.

Se presume que por la situación anterior es que la modelación de los TCE requiere de un mayor cambio en el efecto de aprendizaje para las compras de alimentos por EC, ello supone la verificación de otros factores asociados con los impulsos para efectuar la compra de alimentos en línea los cuales podrían incidir a manera de motivaciones, como por ejemplo, los estímulos asociados con la percepción de la conveniencia de realizar la adquisición de estos bienes por dicho canal.

En tercer lugar, en este trabajo no se encontraron diferencias relevantes en las conductas asociadas a los TCE al agruparse por variables demográficas, lo que sugiere que a futuro se podría profundizar en la verificación de este resultado en virtud de lograr un mejor ajuste del modelo empleado para evaluar la percepción de los TCE que percibe el consumidor.

En cuarto lugar, se logró determinar que 9 de cada 10 compras de alimentos le podría tomar al consumidor menos de 10 minutos en la ejecución de todo el proceso de la toma de decisión, lo cual implica la existencia de ahorros de tiempo importantes en el tiempo total que se debe dedicar para satisfacer la adquisición de estos bienes, esto tomando en cuenta que la proporción existente entre el tiempo que se destina en las compras de alimentos por el principal medio seguido en la actualidad por el consumidor (supermercados) versus el tiempo que se debe emplear en línea para realizar las mismas compras podrá ser hasta de 15:1.

Finalmente se puede decir que la aplicación de ecuaciones estructurales permite una modelación aplicable a otras temáticas económicas de interés para los agronegocios, por lo que en futuros abordajes investigativos se sugiere considerar a esta metodología para el manejo de datos relacionados con percepciones del consumidor de alimentos.

\section{Literatura citada}

Arslan, O., Archetti, C., Jabali, O., Laporte, G., \& Grazia Speranza, M. (2019). Minimum cost network design in strategic alliances. Omega, 102079. https://doi.org/10.1016/j.omega.2019.06.005

Beaujean, A. A. (2014). Latent Variable Modeling Using R: A Step-by-Step Guide. Routledge. https://doi.org/10.4324/9781315869780

Bollen, K. A. (1987). Total, Direct, and Indirect Effects in Structural Equation Models. Sociological Methodology, 17, 37. https://doi.org/10.2307/271028

Cai, S., \& Xu, Y. (2006). Effects of outcome, process and shopping enjoyment on online consumer behaviour. Electronic Commerce Research and Applications, 5(4), 272-281. https://doi. org/10.1016/j.elerap.2006.04.004 
Chan, T. K. H., Cheung, C. M. K., \& Lee, Z. W. Y. (2017). The state of online impulse-buying research: A literature analysis. Information \& Management, 54(2), 204-217. https://doi.org/10.1016/j.im.2016.06.001

Coase, R. H. (1937). The Nature of the Firm. Economica, 4(16), 386-405. https://doi.org/10.1111/j.1468-0335.1937.tbo0002.x

Fang, J., George, B., Shao, Y., \& Wen, C. (2016). Affective and cognitive factors influencing repeat buying in e-commerce. Electronic Commerce Research and Applications, 19, 44-55. https://doi.org/10.1016/j.elerap.2016.08.001

Floh, A., \& Madlberger, M. (2013). The role of atmospheric cues in online impulse-buying behavior. Electronic Commerce Research and Applications, 12(6), 425-439. https://doi.org/10.1016/j.elerap.2013.06.001

Gefen, D. (2000). E-commerce: The role of familiarity and trust. Omega, 28(6), 725-737. https://doi.org/10.1016/S0305-0483(00)00021-9

INEC. (2019). ENAHO | INSTITUTO NACIONAL DE ESTADISTICA Y CENSOS. http://wwW. inec.go.cr/ingresos-y-gastos-de-hogares/ingresos-de-los-hogares

Kacen, J. J., Hess, J. D., \& Kevin Chiang, W.-Y. (2013). Bricks or Clicks? Consumer Attitudes toward Traditional Stores and Online Stores. Global Economics and Management Review, 18(1), 12-21. https://doi.org/10.1016/S2340-1540(13)70003-3

Kim, C., Galliers, R. D., Shin, N., Ryoo, J.-H., \& Kim, J. (2012). Factors influencing Internet shopping value and customer repurchase intention. Electronic Commerce Research and Applications, 11(4), 374-387. https://doi.org/10.1016/j.elerap.2012.04.002

Kline, R. (2011). Principles and practice of structural equation modeling (Tercera Ed.). The Guilford Press.

Liao, C., Liu, C.-C., \& Chen, K. (2011). Examining the impact of privacy, trust and risk perceptions beyond monetary transactions: An integrated model. Electronic Commerce Research and Applications, 10(6), 702-715. https://doi.org/10.1016/j.elerap.2011.07.003

Lin, J., Lu, Y., Wang, B., \& Wei, K. K. (2011). The role of inter-channel trust transfer in establishing mobile commerce trust. Electronic Commerce Research and Applications, 10(6), 615625. https://doi.org/10.1016/j.elerap.2011.07.008

Martínez-López, F. J., Pla-García, C., Gázquez-Abad, J. C., \& Rodríguez-Ardura, I. (2014). Utilitarian motivations in online consumption: Dimensional structure and scales. Electronic Commerce Research and Applications, 13(3), 188-204. https://doi.org/10.1016/j.elerap.2014.02.002 
Pavlou, P. A. (2003). Consumer Acceptance of Electronic Commerce: Integrating Trust and Risk with the Technology Acceptance Model. International Journal of Electronic Commerce, 7(3), 101-134.

PIMA. (2017). Tendencias de Consumo - PIMA. http://Wwww.pima.go.cr/tendencias-de-consumo/

Ramlall, I. (2017). Applied Structural Equation Modelling for Researchers and Practitioners: Using R and Stata for Behavioural Research: Vol. First edition. Emerald Group Publishing Limited. http://search.ebscohost.com/login.aspx?direct=true\&db=e000xww\&AN=1423582\&site=ehost-live\&scope=site

Smith, V. L. (1989). Theory, Experiment and Economics. Journal of Economic Perspectives, 3(1), 151-169. https://doi.org/10.1257/jep.3.1.151

Teo, T. S. H., \& Yu, Y. (2005). Online buying behavior: A transaction cost economics perspective. Omega, 33(5), 451-465. https://doi.org/10.1016/j.omega.2004.06.002

Villalobos-Monge, A., \& Sánchez-Chacón, L. (2013). Caracterización del consumo de hostalizas en las familias del Sur-Sur de Costa Rica. Agronomía Mesoamericana, 24(1), 37-46.

Williamson, O. E. (1989). Chapter 3 Transaction cost economics. En Handbook of Industrial Organization (Vol. 1, pp. 135-182). Elsevier. https://doi.org/10.1016/S1573-448X(89)01006-X

Wright, R., Tekin, E., Topalli, V., McClellan, C., Dickinson, T., \& Rosenfeld, R. (2017). Less Cash, Less Crime: Evidence from the Electronic Benefit Transfer Program. Journal of Law \& Economics, 6o(2), 361-383. https://doi.org/10.1086/693745

Xu-Priour, D. L., Cliquet, G., \& Palmer, A. (2017). The Influence of Buyers' Time Orientation on Online Shopping Behavior: A Typology. International Journal of Electronic Commerce, 21(3), 299-333. https://doi.org/10.1080/10864415.2016.1319206 


\section{Anexos}

Anexo 1. Información demográfica de la población encuestada según porcentaje total, edad y época de compra. $\mathrm{N}=223$

\begin{tabular}{|c|c|c|c|c|c|c|c|c|c|c|}
\hline \multirow{2}{*}{$\begin{array}{c}\text { VARIABLE } \\
\text { DEMOGRAFICA }\end{array}$} & \multirow{2}{*}{$\begin{array}{l}\text { CLASIFICACI } \\
\text { ON }\end{array}$} & \multirow{2}{*}{$\begin{array}{l}\text { PORCENTA } \\
\text { JEDE. } \\
\text { TOTAL }\end{array}$} & \multicolumn{4}{|c|}{ EPOCA SECA } & \multicolumn{4}{|c|}{ EPOCA LLUVIOSA } \\
\hline & & & $\begin{array}{c}\text { Edad } \\
\text { Medi } \\
\mathbf{a}\end{array}$ & $\begin{array}{c}\text { Eda } \\
\text { d } \\
\text { Mtox } \\
.\end{array}$ & $\begin{array}{c}\text { Eda } \\
\text { d } \\
\text { Min } \\
.\end{array}$ & $\begin{array}{c}\text { Eda } \\
\text { d } \\
\text { Des } \\
\text { Y. } \\
\end{array}$ & $\begin{array}{c}\text { Edad } \\
\text { Medi } \\
\text { a }\end{array}$ & $\begin{array}{c}\text { Eda } \\
\text { d } \\
\text { Mtox } \\
.\end{array}$ & $\begin{array}{c}\text { Eda } \\
\text { d } \\
\text { Min } \\
.\end{array}$ & $\begin{array}{c}\text { Eda } \\
\text { d } \\
\text { Des } \\
\text { v. }\end{array}$ \\
\hline \multirow[t]{2}{*}{ Sexo } & Hombre & $17.9 \%$ & 45 & 67 & 28 & 12 & 39 & 80 & 21 & 10 \\
\hline & Mujer & $82.1 \%$ & 42 & 72 & 23 & 10 & 42 & 68 & 23 & 10 \\
\hline \multirow[t]{6}{*}{ Escolaridad } & $\begin{array}{l}\text { Bachillerato } \\
\text { secundaria }\end{array}$ & $12,1 \%$ & 43 & 57 & 23 & 11 & 41 & 81 & 21 & 11 \\
\hline & $\begin{array}{l}\text { Bachillerato } \\
\text { uriversitario }\end{array}$ & $215 \%$ & 35 & 63 & 23 & 11 & 39 & 59 & 25 & 10 \\
\hline & Licenciatura & $29.1 \%$ & 41 & 63 & 27 & 9 & 40 & 57 & 24 & 11 \\
\hline & Maestria & $27.8 \%$ & 47 & 72 & 32 & 10 & 45 & 68 & 28 & 10 \\
\hline & Doctorado & $54 \%$ & 53 & $B 0$ & 49 & 5 & 45 & 55 & 34 & 9 \\
\hline & Otro & $4.0 \%$ & 42 & 81 & 30 & 14 & 50 & 56 & 40 & 9 \\
\hline \multirow[t]{6}{*}{ Ingreso familiar } & $\begin{array}{l}\text { Hasta } \\
500.000 \\
\text { colones/mes }\end{array}$ & $22.9 \%$ & 37 & 50 & 23 & 7 & 40 & 57 & 23 & 10 \\
\hline & $\begin{array}{l}\text { Entre } 501000 \\
y 1000.000 \\
\text { colones/mes }\end{array}$ & $28.7 \%$ & 40 & 81 & 28 & 10 & 41 & 63 & 24 & 11 \\
\hline & $\begin{array}{l}\text { Entre } \\
10 \text { oon y } \\
1500.000 \\
\text { colones/mes }\end{array}$ & $18,1 \%$ & 46 & 63 & 27 & 13 & 38 & 54 & 21 & 9 \\
\hline & $\begin{array}{l}\text { Entre } \\
1.500 .001 y \\
2.000000 \\
\text { colones/mes }\end{array}$ & $143^{\prime} \%$ & 48 & 67 & 32 & 11 & 46 & 65 & 28 & 11 \\
\hline & $\begin{array}{l}\text { Entre } \\
\text { 2.000oni y } \\
3000000 \\
\text { colones/mes }\end{array}$ & $11,7 \%$ & 45 & 72 & 30 & 12 & 47 & 60 & 33 & 9 \\
\hline & $\begin{array}{l}\text { Mas de } \\
3 \text { ono oco } \\
\text { colones/mes }\end{array}$ & $5,3 \%$ & 49 & $6 z$ & 39 & 8 & 44 & 53 & 34 & 8 \\
\hline \multirow[t]{5}{*}{ Miembros por familla } & Vhe soloia: & $135^{1 \%}$ & 42 & 63 & 27 & 11 & 44 & E1 & 30 & 9 \\
\hline & Dos personas & $.37,2 \%$ & 44 & 67 & 27 & 12 & .78 & $6 \overline{1}$ & 23 & 11 \\
\hline & Tres personas & $215 \%$ & 45 & 72 & 23 & 12 & 45 & 6o & 34 & 3 \\
\hline & $\begin{array}{l}\text { Cuatro } \\
\text { personas }\end{array}$ & $20,6 \%$ & 38 & 51 & 23 & 7 & 43 & 60 & 26 & 10 \\
\hline & $\begin{array}{l}\text { Mas de } \\
\text { cuatro } \\
\text { personas }\end{array}$ & $7,2 \%$ & 46 & 48 & 43 & 4 & 43 & 63 & 21 & 12 \\
\hline
\end{tabular}


Anexo 2. Efectos directos, indirectos y totales de las variables empleadas en el modelo propuesto respecto a la respuesta del consumidor de alimentos por EC.(Negrita=significancia menor a 0.05$) \mathrm{N}=223$

\begin{tabular}{|c|c|c|c|}
\hline \multirow[b]{2}{*}{ VARIABLE } & \multicolumn{3}{|c|}{ EFECTO } \\
\hline & DIRECTO & INDIRECTO & TOTAL \\
\hline CF2 & 0,95 & 0,34 & 1,29 \\
\hline $\mathrm{CF}_{3}$ & 0,95 & 0,34 & 1,29 \\
\hline $\mathrm{CF}_{4}$ & 0,97 & 0,35 & 1,32 \\
\hline $\mathrm{CF}_{5}$ & 0,97 & 0,35 & 1,32 \\
\hline HD1 & 0,99 & 0,57 & 1,56 \\
\hline HD2 & 0,99 & 0,06 & 1,04 \\
\hline HD3 & 0,99 & 0,06 & 1,05 \\
\hline $\mathrm{TC}_{1}$ & 0,94 & 0,06 & 0.99 \\
\hline $\mathrm{TC} 2$ & 0,97 & 0,06 & 1,03 \\
\hline $\mathrm{TC}_{3}$ & 0,97 & 0,06 & 1.02 \\
\hline R1 & 0,97 & - & 0,97 \\
\hline R2 & 0,98 & - & 0.98 \\
\hline R3 & 0,96 & - & 0,96 \\
\hline
\end{tabular}


Anexo 3. Visitas por semana, percepción del tiempo promedio empleado por visita en la compra de alimentos según principal canal empleado. $\mathrm{N}=223$

\begin{tabular}{|c|c|c|c|}
\hline RUBRO & SUPERMERCADO & $\begin{array}{l}\text { FERIA DEL } \\
\text { AGRICULTOR }\end{array}$ & INTERNET \\
\hline $\begin{array}{l}\text { Porcentaje de } \\
\text { respuestas } \\
\text { preferencia \#1 }(\%)\end{array}$ & 62.0 & 23.4 & 10.7 \\
\hline $\begin{array}{l}\text { Visitas promedio por } \\
\text { semana (Veces) }\end{array}$ & 3 & 1 & 3 \\
\hline $\begin{array}{l}\text { Promedio del tiempo } \\
\text { empleado por visita } \\
\text { (minutos) }\end{array}$ & $52.8 \pm 45-55$ & $42.24 \pm 50.99$ & $15.74 \pm 44.41$ \\
\hline $\begin{array}{l}\text { Mediana del tiempo } \\
\text { empleado por visita } \\
\text { (minutos) }\end{array}$ & 45 & 30 & 3 \\
\hline $\begin{array}{l}\text { Máximo del tiempo } \\
\text { empleado por visita } \\
\text { (minutos) }\end{array}$ & 240 & 240 & 480 \\
\hline $\begin{array}{l}\text { Mínimo del tiempo } \\
\text { empleado por visita } \\
\text { (minutos) }\end{array}$ & 1 & 5 & 2 \\
\hline $\begin{array}{l}\text { Tiempo total } \\
\text { empleado en las } \\
\text { compras } \\
\text { alimentos } \\
\text { (minutos/semana) }\end{array}$ & $\begin{array}{l}158.4^{1 /} \\
135^{2 /}\end{array}$ & $\begin{array}{c}42.24^{1 /} \\
30^{2 /}\end{array}$ & $\begin{array}{c}47.22^{2 /} \\
9^{2 /}\end{array}$ \\
\hline
\end{tabular}

1/Tiempo total considerando el promedio de minutos empleados en cada compra multiplicado por la cantidad promedio de visitas semanales

2/Tiempo total considerando la mediana de minutos empleados en cada compra multiplicado por la cantidad promedio de visitas semanales 
Anexo 4. Estadísticas de los minutos por compra según sexo efectuadas por el sitio Agroferia UCR. Febrero-Noviembre 2019. $\mathrm{N}=1813$

\begin{tabular}{ccc}
\hline PARAMETRO & HOMBRES & MUJERES \\
\hline $\begin{array}{c}\text { Recuento (cantidad de } \\
\text { compras) }\end{array}$ & 318 & 1495 \\
$\begin{array}{c}\text { Promedio } \pm \text { Desviación } \\
\text { estándar }\end{array}$ & $0: 04: 25,15 \pm 0: 07: 17,98$ & $0: 04: 04,71 \pm 0: 05: 48,87$ \\
Mediana & $0: 01: 59,00$ & $0: 02: 11,00$ \\
Moda & $0: 00: 42,99$ & $0: 00: 54,00$ \\
Máximo & $0: 57: 51,00$ & $0: 58: 38,99$ \\
Mínimo & $0: 00: 19,00$ & $0: 00: 09,99$ \\
\hline
\end{tabular}

